\title{
Evaluación de los estilos de aprendizaje y enseñanza en estudiantes y docentes
}

Assessment of learning and teaching styles in students and teachers

\section{Avaliação de estilos de aprendizagem e ensino em}

\section{estudantes e professores}

\author{
Adriana Aponte Rivera*, Jeimmy Nathalia Arévalo Morales, Camila Andrea \\ Calderón Ramírez, Paula Andrea Rodríguez Clavijo, \& Zulma Clareth Salamanca Duarte
}

Univesidad Incca de Colombia

\section{Citar este artículo así:}

Aponte, A., Arévalo, J. N., Calderón, C. A., Rodríguez, P. A., \& Salamanca, Z. C. (2020). Evaluación de los estilos de aprendizaje y enseñanza en estudiantes y docentes. Revista Enfoques, 3(1), 61-9o. http://dx.doi.org/

Derechos de autor: Licencia Creative Commons AtribuciónNoComercial-SinDerivadas 4.0 internacional y 2.5 Colombia (CC BYNC-ND 2.5 (O)

Recibido: agosto 17 de 2018

Revisado: octubre 03 de 2018

Aceptado: noviembre 07 de 2019

* Contacto: Adriana Aponte Rivera: adrianaponte519@yahoo.es ORCID: 0000-0002-2271-4480 
Resumen | La presente investigación surgió con base en las problemáticas observadas en el contexto educativo evidenciando que las dificultades más comunes dentro de la población estudiantil son la baja motivación, la deserción escolar y el bajo rendimiento académico. Dichas dificultades, están estrechamente ligadas a que no se tiene un conocimiento previo de los estilos de aprendizaje y de enseñanza propios de cada individuo. Por tal motivo, el objetivo principal de esta investigación consistió en la evaluación e identificación de los estilos de aprendizaje y de enseñanza en estudiantes y docentes del colegio Unión Europea, con el propósito de establecer una posible relación entre los estilos; la evaluación se realizó por medio de la aplicación de los cuestionarios CHAEA Junior y CEE. Metodológicamente esta investigación se llevó a cabo desde el enfoque cuantitativo, con un diseño de tipo transversal-descriptivocomparativo. La población que participó en este estudio fueron los estudiantes y docentes del colegio Unión Europea y María Cano, de los cuales se seleccionó una muestra de estudiantes de los grados $4^{\circ}$ a $7^{\circ}$ con sus respectivos directores de curso. La muestra fue seleccionada por medio de un muestreo probabilístico de tipo estratificado. Los resultados obtenidos evidencian que en el total de la muestra no existe una relación significativa entre los estilos de enseñanza implementados por los docentes en el aula y los estilos propios del alumnado al momento de adquirir nuevos conocimientos, sin embargo, se evidenció que en tres de los grados evaluados si existe una correlación entre dichos estilos.

Palabras clave / Evaluación, estilos, aprendizaje, enseñanza.

Abstract | The present investigation arose based on the problems observed in the educational context, showing that the most common difficulties within the student population are low motivation, school dropout and low academic performance, etc., these difficulties are closely linked to the fact that there is prior knowledge of the learning and teaching styles of each individual. For this reason, the main objective of this research was the evaluation and identification of learning and teaching styles in students and teachers of the European Union school, with the purpose of establishing a possible relationship between the styles; The evaluation was carried out through the application of the CHAEA Junior and CEE questionnaires. Methodologically, this research was carried out from the quantitative approach, with a cross-descriptive-comparative design. The population that participated in this study were the students and teachers of the European Union and María Cano school, from which a sample of students from grades 4 to 7 with their respective course directors was selected, said sample 
was selected through of a probabilistic sampling of stratified type. The results obtained show that in the total sample there is no significant relationship between the teaching styles implemented by teachers in the classroom and the students' own styles when acquiring new knowledge, however, it was evidenced that in three of the grades evaluated if there is a correlation between these styles.

Keywords / Evaluation, styles, learning, teaching.

Resumo | A presente investigação surgiu com base nos problemas observados no contexto educacional, mostrando que as dificuldades mais comuns na população estudantil são baixa motivação, abandono escolar e baixo desempenho acadêmico etc., essas dificuldades estão intimamente ligadas ao fato de existirem conhecimento dos estilos de aprendizagem e ensino de cada indivíduo. Por esse motivo, o principal objetivo desta pesquisa foi a avaliação e identificação de estilos de aprendizagem e ensino em alunos e professores da escola da União Europeia, com o objetivo de estabelecer uma possível relação entre os estilos; A avaliação foi realizada através da aplicação dos questionários CHAEA Junior e CEE. Metodologicamente, esta pesquisa foi realizada a partir da abordagem quantitativa, com delineamento descritivo-comparativo. A população que participou deste estudo foram os alunos e professores da União Europeia e da escola María Cano, da qual foi selecionada uma amostra de alunos das séries 4 a 7 com seus respectivos diretores de cursos, sendo essa amostra selecionada por meio de uma amostra probabilística de tipo estratificado. Os resultados obtidos mostram que, na amostra total, não existe relação significativa entre os estilos de ensino implementados pelos professores em sala de aula e os próprios estilos dos alunos na aquisição de novos conhecimentos; no entanto, evidenciou-se que em três das séries avaliadas se existe uma correlação entre esses estilos.

Palavras-chave / Avaliação, estilos, aprendizagem, ensino.

\section{Introducción}

La presente investigación se desarrolló con el propósito de evaluar los diferentes estilos de aprendizaje y de enseñanza presentes en estudiantes y docentes de una institución educativa de la ciudad de Bogotá, dicha evaluación, se llevó a cabo por medio de los cuestionarios Honey-Alonso de estilos de aprendizaje (CHAEA) publicado en el año (1997) y el cuestionario de estilos de enseñanza (CEE) de Martínez-Geijo del año (2001). Este estudio se realizó en el Colegio Unión Europea, el cual se encuentra situado en la localidad de Ciudad Bolívar, UPZ 19, en el sector de Lucero Medio, esta institución es de carácter público la cual cuenta con dos sedes institucionales (sede Unión Europea y sede María Cano), conformada por población 
mixta en la jornada mañana y tarde. Esta investigación se implementó específicamente con la población estudiantil de los grados $4^{\circ}$ a $7^{\circ}$ de la jornada tarde, de igual manera se incluyó una muestra conformada por los docentes directores de dichos cursos.

Se realizó una revisión teórica acerca de los estilos de aprendizaje y enseñanza en el contexto educativo; encontrándose a Donado 2014 quien define que el estilo de aprendizaje del estudiante está relacionado con aspectos cognitivos, afectivos y fisiológicos que impactan en todas las dimensiones del ser humano y que constituye una herramienta importante para la formación integral de los estudiantes, del mismo modo, son relevantes aquellos factores de orden biológico o sociocultural, como el sexo, la edad, las primeras experiencias educativas, el papel de los padres en su educación, el estrato socioeconómico y otra serie de elementos que afectan las expectativas personales y profesionales como el rendimiento académico, las estrategias de estudio, la elección profesional y la autoestima, etc. (Donado, 2014).

Por otra parte, Renes, Echeverry, Chiang \& Rangel (2013) definen los estilos de enseñanza como características personales y de comportamiento que favorecen la enseñanza que el docente implementa habitualmente en cada proceso de aprendizaje, dichas características son adquiridas a través de su experiencia académica y profesional, teniendo como referente sus propios estilos de aprendizaje. Por consiguiente, Laudadío \& Da Dalt (2014) establecen cinco factores que determinan el modo de enseñar de los docentes. En primer lugar, se encuentran las capacidades de los estudiantes tales como los conocimientos previos, iniciativa, motivación, responsabilidad y madurez. En segundo lugar, las necesidades del docente respecto al control de la clase en torno a la organización, presentación de metas por alcanzar, seguimiento del progreso de los estudiantes, determinación o evaluación del nivel de rendimiento. Posteriormente, la capacidad del profesor para construir y mantener una relación educativa teniendo en cuenta la comunicación bidireccional, escucha atenta, habilidades de comunicación interpersonal; de igual manera las estrategias de enseñanza y por ultimo las demandas de la situación o del contexto (Laudadío \& Da Dalt, 2014).

Esta investigación se realizó con el fin de evaluar los estilos de aprendizaje en estudiantes y estilos de enseñanza en docentes del colegio Unión Europea; teniendo en cuenta que los estilos propios de cada individuo al momento de aprender hacen parte fundamental del ser humano y dichos estilos están relacionados con los estilos de enseñanza que favorecen los 
procesos de adquisición de nuevos conocimientos. Son diversos los rasgos que caracterizan la forma en que cada persona aprende, lo cual exige especificar el modo en que las personas acceden al conocimiento con el fin de garantizar un proceso de enseñanza que contribuya a mejorar los aspectos cognitivos, emocionales, fisiológicos y contextuales de las personas.

Por tal motivo, surgió la necesidad de comprender e identificar los estilos de aprendizaje y su relación con los estilos de enseñanza, debido a que en el contexto educativo es importante esclarecer dicha relación dado que esto permita generar estrategias que potencialicen los procesos de aprendizaje. En esencia el propósito de esta investigación se enfocó en evaluar e identificar la relación existente entre los estilos de aprendizaje de un grupo de estudiantes y los estilos de enseñanza de sus profesores (García, Ortiz \& Rodríguez, 2016).

Teniendo en cuenta la investigación que realizó Delgado (2014), se determinó que el estilo predominante en la población estudiantil es el reflexivo con un 37,3\%. Según este autor estos alumnos responden de manera adecuada a las exigencias propias de la educación tradicional; además, esta investigación permitió conocer y evaluar los estilos de enseñanza utilizados en el aula y así mismo determinar si estas estrategias utilizadas por los profesores favorecen la adquisición y construcción del conocimiento en los estudiantes; para de esta manera establecer si existe una relación entre los estilos de enseñanza utilizados por los profesores y los estilos de aprendizaje implementados por los alumnos; permitiendo reconocer la construcción de hábitos y estrategias de estudio, que favorecen o por el contrario pueden afectar el aprendizaje y así mismo el rendimiento académico (pp.192).

De acuerdo a lo estipulado en el Decreto 1290 de 2009 que tiene como objetivo reglamentar la evaluación del aprendizaje y promoción de los estudiantes de los niveles de educación básica y media que deben realizar los establecimientos educativos. Además, exige por parte del docente retomar nuevas y mejores estrategias para la evaluación rompiendo de esta manera el paradigma de lo tradicional fomentado el cambio. Lo importante a tener en cuenta de este decreto es su flexibilidad en cuanto a su aplicación ya que es una herramienta que facilitará el desarrollo, capacidades y habilidades, de los estudiantes, contribuyendo a identificar su estilo de aprendizaje permitiendo su formación integral. (Colombia, Ministerio de Educación Nacional, 2009). 


\section{El presente estudio}

El aprendizaje es un proceso fundamental en la formación del ser humano quien está en una constante interacción con el mundo que le rodea; dicha interacción engloba factores como el medio ambiente, la familia, la sociedad y la escuela que se convierte en un elemento determinante en la construcción de su proyecto de vida. Partiendo de lo anterior, se hace necesario, el conocimiento del estilo de enseñanza que ejercen los docentes en los ambientes escolares para, de este modo, determinar la relación con el estilo de aprendizaje propio de cada alumno, que permita identificar la forma de aprender y adquirir conocimientos, cumpliendo con el propósito que tienen los centros educativos de formación que consiste en que el estudiante realmente aprenda y adquiera todas las habilidades personales y profesionales que favorezcan su desarrollo intelectual y personal. Teniendo en cuenta la problemática observada en la documentación de seguimiento escolar dentro de los parámetros del comité académico establecidos por el contexto educativo Colegio Unión Europea se logró evidenciar que las dificultades más comunes en los estudiantes son la baja motivación, deserción escolar, bajo rendimiento académico y problemas de convivencia. Dichas dificultades están estrechamente ligadas a que no se tiene un conocimiento previo de los estilos de aprendizaje y de enseñanza propio de cada individuo. Por tal motivo, se hizo necesario evaluar e identificar los estilos por medio de los cuestionarios CHAEA Junior y CEE; con el propósito, de garantizar un mejor proceso educativo que transforme la educación tradicional en torno a una visión multidimensional de los estilos de enseñanza y de aprendizaje promoviendo así una educación incluyente.

De igual manera, es necesario conocer el entorno psicológico en el que se desarrolla el estudiante durante su proceso de aprendizaje, para evaluar y determinar los estilos de aprendizaje y enseñanza mejorando las condiciones en las que se está produciendo el desarrollo y previniendo problemas futuros en su educación. Dicha necesidad surge dada la importancia de incrementar la calidad educativa buscando prestar atención al mejoramiento de los procesos que se dan dentro del aula; por ello, se debe tener en cuenta que la motivación está relacionada intrínsecamente con el clima educativo para contribuir de manera activa y dinámica con los procesos de enseñanza y aprendizaje, el alumno necesita una fuerte motivación para aprender y desarrollar conocimientos, actitudes y estrategias que le permitan enfrentarse al mundo académico, al entorno social y posteriormente a la vida laboral. Por consiguiente, el docente debe rescatar pautas de acción que incorporen a la motivación 
como un aspecto significativo de su práctica centrando la atención en el conocimiento y aplicación de estrategias para promover actitudes positivas hacia el aprendizaje (Hernández \& González, 2015).

Según la última Encuesta Nacional de Deserción 2012 del Ministerio de educación, que entrevistó a 46.285 estudiantes, reveló que a medida que avanzaban los cursos, el interés de los alumnos por las materias disminuía. En quinto grado, por ejemplo, el 38,5\% respondió que las clases les parecían divertidas, mientras que en once el porcentaje disminuyó a un 14,4 \%. El mismo informe mostró que el $16 \%$ de los encuestados no consideraba útiles para el futuro las clases que recibían, un factor que ligaban a las causales de deserción. Pese a los avances sostenidos que en materia de retención escolar muestra el país en la última década, en el 2014, de acuerdo con el Ministerio de Educación, cerca de 319 mil niños y adolescentes el $3,07 \%$ del total de la matrícula nacional, que es de 10'381.403, desertaron de sus colegios. La Fundación Dividendo por Colombia coincide en que la deserción afecta más a los escolares de secundaria. Cifras de Mineducación reportan un porcentaje de permanencia en primaria del $96 \%$, mientras que en secundaria apenas alcanza el 67 \% (Sánchez, 2016, p. 1)

Del mismo modo, Freiberg, Ledesma \& Fernández (2015) definen que los estudiantes deben ajustar sus estilos de aprendizaje en el ámbito académico a las formas de conocimiento privilegiadas en la institución, puesto que las orientaciones varían en cuanto a estructura de conocimientos, tecnologías y productos, criterios de excelencia académica, métodos de enseñanza, de evaluación y de investigación. El sistema educativo pauta de antemano una serie de reglas que determinan las habilidades, competencias e intereses que el estudiante debe poseer y desarrollar. Los estilos presentes en los alumnos intervienen en su adaptación académica y se configuran mediatizados por múltiples factores tales como sexo, edad, orientación de la educación recibida, nivel educativo y profesión de los padres, entre otros; todos estos aspectos se tornan importantes a la hora de conocer la versatilidad con la que el alumno logrará adecuar sus estilos predominantes al requerido por la institución, se espera así que estudiantes con estilos diferentes a los requeridos por una orientación modifiquen sus preferencias para aprender a medida que avanzan en sus trayectos académicos (Freiberg, Ledesma \& Fernández, 2015). 


\section{Referentes teóricos}

A lo largo de esta investigación se expondrán los principales fundamentos teóricos relacionados con la evaluación de los estilos de aprendizaje en los estudiantes y de los estilos de enseñanza en los docentes del colegio Unión Europea. Por esta razón, se define, en primera instancia, la psicología educativa, sus características y enfoques orientadores tales como el cognitivo conductual y el constructivismo; posteriormente se definen los conceptos de aprendizaje y enseñanza junto con sus diferentes tipos y características y finalmente se establecieron los principales modelos y estilos de aprendizaje y de enseñanza.

En primera instancia se logró establecer que la psicología educativa es vista como un campo de la psicología que utiliza como método la orientación; debido a que busca guiar a la población infantil y adolescente en sus diferentes procesos del desarrollo emocional, cognitivo, psico-afectivo junto con el establecimiento de relaciones interpersonales en el ámbito académico; aunque estos no son los únicos actores también se incluye el trabajo con docentes, familias y pares. Por consiguiente, la psicología educativa también se enfoca en estudiar y evaluar el aprendizaje humano en especial en instituciones educativas formales; de esta manera, se logran identificar los diferentes estadios de madurez por los que pasa el ser humano en la infancia y adolescencia a lo largo de su desarrollo físico, cognitivo, social y afectivo (Bustos, 2015).

Según Greca \& Moreira (2016) resaltan que uno de los principales autores que contribuyen a la psicología educativa es Piaget (1981), con su teoría de los cuatro estadios de desarrollo infantil, donde el punto más alto es llegar a obtener un pensamiento lógicoabstracto, esto se genera cuando el menor supera un poco más de los 11 años; por lo tanto Piaget se enfoca en entender el desarrollo a nivel cognitivo del menor y a su vez las diferentes etapas por las cuales este supera y como estos van retroalimentando y fortaleciendo sus procesos de aprendizaje, de igual manera establece que no todos los niños van a tener el mismo nivel desarrollo cognitivo generando un leve retroceso en sus procesos, sin embargo, aclara que adquirir ciertos aprendizajes como lo es el lenguaje es más fácil en cierto momento, sin embargo eso se va fortaleciendo a medida que el menor avanza en su desarrollo (Greca \& Moreira, 2016). 
Por otra parte, Rodríguez (2015) destaca que Vygotsky (1999), postuló la teoría sociocultural, que consiste en que los menores desarrollan su aprendizaje mediante la interacción social, la cual permite la adquisición de nuevas habilidades cognitivas que potencializan su desarrollo; de igual modo enmarca la importancia de los progenitores quienes realizan una orientación en sus procesos llegando a generar que el niño interiorice y trasforme su aprendizaje según su contexto. Una vez el niño realice dichos procesos podrá determinar lo que puede llegar a hacer y lo que en el momento no puede conseguir por sí mismo, es decir va logrando discriminar ciertos comportamientos y conductas propias de su edad y a su vez del contexto en el que se encuentra y como lo puede trasformar por medio de la interiorización del aprendizaje (Rodríguez, 2015).

El enfoque cognitivo conductual es visto en la psicología educativa como un modelo que aporta a regular los procesos psicológicos básicos y superiores, los cuales fomentan el almacenamiento de información, sustancialmente al paradigma cognitivo, supone cierta organización de la estructura cognitiva, que está claramente en contra de una visión meramente acumulativa; de igual manera el sujeto es quien elabora las representaciones y las entidades mentales y son estas representaciones las que determinan las formas de actividad subjetiva, es decir que la conducta está regulada y regida por ellas y no por el ambiente externo. En general, se admite que todo el paradigma cognitivo se caracteriza por otorgar cierta preponderancia al sujeto en el acto del conocimiento y se inserta en la tradición racionalista, y a su vez realiza una trasformación de esta (Otero, 2016).

Del mismo modo, en el enfoque cognitivo conductual, el aprendizaje es visto como una actividad exclusiva vinculada al pensamiento humano, a las facultades de conocer, representar, relacionar, transmitir y ejecutar acciones, asociado a esto el aprendizaje es visto de distintas maneras puesto que puede ser como una adquisición de respuestas, o de conocimiento y por último como una construcción de significados. Por otra parte, la psicología educativa emplea diversas técnicas que logran generar herramientas y aportes positivos al desarrollo del menor, entre dichas técnicas se encuentran la economía de fichas, modelamiento y restructuración cognitiva las cuales se expondrán a continuación (Zapata, 2015).

Por consiguiente, Rivera (2016) indica que el constructivismo puede entenderse como una epistemología, es decir como un sistema teórico en el que el conocimiento se obtiene 
mediante un proceso de construcción propia del ser humano. Se expresa como óptica educativa que plantea que el desarrollo del individuo tiene lugar producto de las relaciones sociales que distinguen a la actividad humana, en el caso que nos ocupa las relaciones didácticas, lo que garantiza la asimilación de un tipo particular de experiencia, la histórico-social (p.p. 3-4).

Por ende Díaz \& Hernández (2015) refieren que la postura constructivista se alimenta de las aportaciones de diversas corrientes psicológicas asociadas genéricamente a la psicología cognitiva, el enfoque psicotécnico piagetiano, la teoría de los esquemas cognitivos, la teoría de la asimilación y el aprendizaje significativo, y por último la psicología sociocultural Vygotskiana; puesto que estos autores comparten el principio de la importancia de la actividad constructiva del alumno en la realización de los aprendizajes escolares. El constructivismo es un enfoque que postula la prevalencia de los procesos activos en la construcción del conocimiento, esta habla del sujeto como un ente cognitivo operante, en relación con la interacción del comportamiento y el aprendizaje, lo cual hace énfasis en los mecanismos de influencia sociocultural y en una educación formal (Díaz \& Hernández, 2015).

Sin embargo, Caira, Urdaneta, \& Mata (2014) indican que el aprendizaje se basa en el conocimiento que cada ser humano implementa, este proceso se puede derivar en tres aspectos: producto, este concepto refiere a el conocimiento adquirido; proceso, se refiere a los hechos ocurridos en el transcurso de implementar dicho conocimiento; función, que atribuye a la estimulación de adquirir información, la retención de la misma y el cambio en la conducta. El estudiante genera vínculos entre lo que aprende y sus antiguos conocimientos, para crear su propia comprensión a través del interés que tenga en adquirir dicha información por medio de la percepción y el proceso. Un docente en este caso desde un punto de vista constructivista actúa como mediador entre el estudiante y la información, lo ideal es que este genere situaciones en que el estudiante se mantenga activo de tal manera que en el alumno se generen dudas y sienta la necesidad de adquirir más información, otras maneras de aprender que complazcan sus ideales, aquellos que son conformados por la relación con su entorno social y natural (Caira, Urdaneta, \& Mata Guevara, 2014).

Algunos autores como Canto, Pérez \& Plata (2016) coinciden en que los estilos de aprendizaje son aprendidos en un individuo desde etapas muy tempranas además mencionan que existen algunas teorías que han trabajado y desarrollado el tema sobre la forma de 
aprender, es decir, de los estilos de aprendizaje que son aquellos rasgos cognitivos, afectivos y fisiológicos que sirven como indicadores de cómo los alumnos perciben interacciones y responden a sus ambientes de aprendizaje. Lo anterior se puede relacionar con los modelos existentes sobre estilos de aprendizaje los cuales ofrecen un marco conceptual para entender los comportamientos observados en el aula, y brindan una explicación sobre la relación de dichos comportamientos con la forma en que están aprendiendo los alumnos y, el tipo de estrategias de enseñanza que pueden resultar más eficaces en un momento determinado, ya sea por el contendido temático en si o bien, por las diversas interacciones sociales que se desarrollan en el aula. En primer lugar, la teoría de Kolb (1984) plantea que este estilo se basa en los modelos de aprendizaje basados en la experiencia ya que es fundamental para que el individuo pueda desarrollar observaciones, análisis y reflexiones en la búsqueda de un aprendizaje significativo (Canto, Pérez \& Plata, 2016).

Posteriormente Rangel, Ortiz, \& Rodríguez (2014) señalan que una de las características del aprendizaje es la transformación que se efectúa en la conducta del individuo después de haber adquirido dicho conocimiento, lo cual ha sucedido a través de la experiencia y la práctica que puede manifestare en acciones futuras; también plantean que el aprendizaje se desarrolla cuando el ser humano adquiere nuevas habilidades y se incorporan nuevos conocimientos, a partir de esto se puede determinar la influencia de los estilos en la forma de aprender; cada sujeto puede poseer varios estilos de aprendizaje sin embargo hay uno que prevalecerá en determinadas situaciones. Por una parte se entiende que la conducta está influenciada por la personalidad y que cuando este comportamiento afecta el aprendizaje se está reflejando un estilo de aprendizaje especifico, es necesario aclarar que los estilos de aprendizaje son flexibles y pueden modificarse al pasar el tiempo y dependiendo las situaciones a las que se enfrente cada alumno, a partir de estos se pueden destacar dos factores que determinan la forma de aprender, que son la percepción y el procesamiento de información (Rangel, Ortiz, \& Rodríguez, 2014).

Por consiguiente, García (2016) destaca un predominio en los estilos de aprendizaje en las diferentes ramas de estudio y en cada una de las personas, De igual manera Chiang, Larenas \& Pizarro (2016) indican que existen distintos estilos de enseñanza que relacionan recíprocamente con los estilos de aprendizaje; a continuación, dichos estilos se resumirán en el siguiente cuadro: 
Volumen 3 • Número 1 • enero - junio 2020 • Págs. 61 - 90 •

ISSN: 2389-8798 impreso / ISSN 2539-1445 (En línea) / Tunja, Boyacá (Colombia)

Tabla 1 | Resumen de los estilos de enseñanza y aprendizaje

\section{Estilos de enseñanza \\ Estilos de aprendizaje}

Abierto: Aquellos docentes que con sus comportamientos de enseñanza favorecen a los alumnos con estilo de aprendizaje activo, los docentes de este estilo se plantean con frecuencia nuevos contenidos, motivan con actividades novedosas y/o con problemas reales del entorno.
Activo: Las personas que tienen predominancia en este estilo se implican totalmente y sin prejuicios en las experiencias, son de mente abierta y realizan con entusiasmo tareas nuevas; prefieren dialogar, les gusta dirigir debates, o realizar presentaciones.
Formal: Son docentes que facilitan a los estudiantes con estilo de aprendizaje reflexivo, los docentes de este estilo son partidarios de la planificación detallada; no admiten la improvisación y no suelen impartir contenidos que no estén incluidos en el programa.
Reflexivo: A las personas reflexivas les gusta considerar experiencias y observarlas desde diferentes perspectivas; reúnen datos, analizándolos con detenimiento antes de llegar a alguna conclusión.
Estructurado: Docentes que ayudan a los alumnos con estilo de aprendizaje teórico, los docentes de este estilo otorgan importancia a la planificación y ponen énfasis en que sea coherente, estructurada y bien presentada. con estilo de aprendizaje pragmático, los docentes de este estilo de enseñanza se preocupan de cómo llevar la planificación a la práctica, las explicaciones son breves y siempre incluyen ejemplos prácticos

Funcional: Estos docentes benefician a estudiantes son breves y siempre incluyen ejemplos prácticos
Teórico: Las personas que son teóricas enfocan los problemas por etapas lógicas, tienden a ser perfeccionistas, integran los hechos en teorías coherentes; son profundos a la hora de establecer teorías, principios y modelos, les gusta analizar y sintetizar, además buscan la racionalidad y la objetividad.
Pragmático: Las personas pragmáticas buscan la aplicación práctica de las ideas y aprovechan la primera oportunidad para experimentarlas, buscan una mejor manera para hacer las cosas, toman decisiones de forma rápida y les gusta resolver problemas.

Fuente: Elaboración propia. 
Por otra parte, Peiteado (2013) indica que el profesor es el encargado de crear el ambiente educativo adecuado y de controlar el proceso de enseñanza mediante técnicas y métodos de instrucción para el alumno, el docente debe tener en cuenta que el objetivo principal en la enseñanza debe cubrir las necesidades de aprendizaje de cada estilo; por tal motivo para que el alumno se interese en aprender, el educador debe tomar, como objetivos de enseñanza, principios que sean compatibles con las preferencias e intereses en relación al estilo de aprendizaje. Es necesario que los profesores conozcan su propio estilo de enseñanza como base para el desarrollo de estrategias de enseñanza y aprendizaje; esto les permitirá considerar otros estilos de aprendizaje diferentes al preferido por él mismo, así como mejorar su estilo de enseñanza particular y complementarlo con otros, para asumir desde la educación, la importancia de incorporar los estilos de aprendizaje, permite llegar a diversos grupos de estudiantes, de diferente edad, nacionalidad y cultura; además aumenta la garantía de los sistemas de enseñanza, favoreciendo una mayor retención de la información por parte de los alumnos (Peiteado, 2013).

Finalmente, García, Ortiz \& Rodríguez (2016) definieron cinco estilos de enseñanza específicos: el primero se cataloga como experto y es aquel profesor que por su conocimiento detallado de la disciplina que imparte mantiene un estatus y reta a sus alumnos mediante la competencia. El segundo el de autoridad formal, al igual que el anterior tiene un conocimiento estructurado de su disciplina y mantiene un estatus entre sus alumnos, pero a su vez es ordenado, hace retroalimentación del conocimiento impartido y se apoya en la normatividad institucional. El tercero es el modelo personal, donde el profesor se cree ejemplo para sus estudiantes y a través de su desempeño meticuloso y ordenado, muestra a sus alumnos la manera adecuada de pensar y comportarse; el cuarto es el facilitador, en el cual el profesor se identifica como asesor en el proceso del aprendizaje de los estudiantes, usando el cuestionamiento, llevándolos hacia la autonomía y la responsabilidad. Por último, es el delegador, un profesor que se identifica como consultor, que da libertad al estudiante para que trabaje proyectos de manera independiente o en equipo. Sin embargo, estos estilos pueden variar según el grupo de estudiantes o la clase impartida, incluso a través del tiempo, el maestro es capaz de ir cambiando su estilo de enseñanza, producto de su vivencia y su práctica (García, Ortiz \& Rodríguez, 2016) 


\section{Método}

\section{Tipo de estudio y diseño}

La investigación se llevó a cabo desde el enfoque cuantitativo caracterizado por ser secuencial y probatorio, fundamentado en un conjunto de procesos como la recolección de datos para probar hipótesis, con base en la medición numérica y el análisis estadístico de las variables, con el fin de establecer patrones de comportamiento y probar teorías. En una investigación cuantitativa se pretende generalizar los resultados encontrados en un grupo o segmento (muestra) a una colectividad mayor (universo o población); también se busca que los estudios efectuados puedan replicarse, de igual manera con este tipo de estudio se intenta explicar y predecir los fenómenos investigados, buscando regularidades y relaciones causales entre variables, esto significa que el propósito principal es la construcción y demostración de teorías. Para este enfoque, si se sigue rigurosamente el proceso y de acuerdo con ciertas reglas lógicas, los datos generados poseen los estándares de validez y confiabilidad y las conclusiones derivadas contribuirán a la generación de conocimiento. El diseño de esta investigación es de tipo transversal-descriptivo-comparativo, el cual mide la prevalencia o incidencia en una muestra poblacional en un solo momento temporal (Hernández Sampieri, Fernández Collado \& Baptista Lucio, 2010).

\section{Participantes}

La población que participó en este estudio fueron los estudiantes y docentes de primaria y secundaria del colegio Unión Europea y María Cano, de los cuales se seleccionó una muestra de estudiantes de los grados $4^{\circ}$ a $7^{\circ}$ con sus respectivos directores de curso; dicha muestra fue seleccionada por medio de un muestreo probabilístico de tipo estratificado el cual consiste en la selección de conjuntos o subconjuntos de sujetos de una población que presenten una característica determinada que genere un interés particular para el estudio, en este caso la característica principal fue el estilo de aprendizaje y de enseñanza de cada participante; el tamaño de la muestra se estableció según el número de estudiantes que conforman cada curso y la muestra de docentes se determinó de acuerdo al número de cursos evaluados (García, 2009). 


\section{Instrumentos}

Los instrumentos implementados para la evaluación e identificación de los estilos de aprendizaje y de enseñanza fueron el cuestionario CHAEA Junior y el CEE. El cuestionario CHAEA Junior, es un instrumento que parte en base a los fundamentos teóricos de Kolb (1984), desde la premisa de que el modelo de aprendizaje por experiencia favorece el mejoramiento del rendimiento académico. Este cuestionario permite identificar el estilo de aprendizaje principal que presenta cada estudiante; además, determinar el perfil preferente de estilo de aprendizaje en los estudiantes; dichos perfiles son concebidos y entendidos desde una concepción cíclica, y teniendo en cuenta las características psicológicas y cronológicas que presentan los niños de esta edad. El cuestionario consta de 44 premisas, divididos en cuatro grupos de 11 ítems, y al igual que la versión original (CHAEA), estos grupos corresponden a cada uno de los cuatro estilos de aprendizaje, el activo, el reflexivo, el teórico y el pragmático; con respecto a los puntajes de calificación en este instrumento, la puntuación absoluta obtenida en cada categoría debe ser como máximo de 11 puntos (Delgado, 2014).

Por otra parte, el cuestionario de estilos de enseñanza (CEE) propuesto por MartínezGeijo (2001), el cual se desarrolló en función de los estilos de aprendizaje planteados por HoneyAlonso, fue diseñado con el fin de identificar el perfil de los estilos de enseñanza presentes en los profesores, este instrumento consta de 80 ítems que pretenden determinar el tipo de enseñanza que desarrolla un docente en su discurso durante las clases, clasificándolo como un estilo abierto, formal, estructurado o funcional; cada uno de estos estilos de enseñanza, presenta características relacionadas con los estilos de aprendizaje de los estudiantes; para su aplicación, las afirmaciones deben ser respondidas con un signo positivo (+) si se está en de acuerdo, con un signo negativo (-) si se está en desacuerdo, dentro de este instrumento no se encuentran respuestas correctas o incorrectas, y para establecer los resultados, estos se obtienen tras la agrupación de las respuestas de acuerdo a las características de cada uno de los estilos de enseñanza (Salgado, Díaz \& Rivas, 2013).

\section{Procedimiento}

Para el desarrollo de la misma se realizó solo una única aplicación de los instrumentos de evaluación en un momento específico del tiempo. Por ende, lo descriptivo se vio reflejado en el análisis de la incidencia de variables en la población elegida, puesto que se realizó la 
descripción de los estilos de enseñanza y aprendizaje presentes en la población. Posteriormente se compararon las variables, para analizar la posible relación existente entre los estilos de enseñanza implementados por los docentes en el aula y los estilos propios de los estudiantes al momento de adquirir nuevos conocimientos que favorezcan y potencialicen los procesos académicos y personales

\section{Resultados}

A continuación, se expondrán los resultados obtenidos de la aplicación de los instrumentos CHAEA Junior y CEE, los cuales reflejan los estilos predominantes dentro de la población participante, dichos resultados fueron analizados estadísticamente y serán presentados por medio de la tabulación y gráficas de manera general e individual correspondiente a cada uno de los grados evaluados.

Tabla 2 | Estilos de aprendizaje de los estudiantes del Colegio Unión Europea Sede A y B Grados $4^{\circ}$ a $7^{\circ}$

\begin{tabular}{lc}
\hline ESTILO & \# ESTUDIANTES \\
\hline ACTIVO & 19 \\
\hline REFLEXIVO & 136 \\
\hline TEORICO & 77 \\
\hline PRAGMATICO & 33 \\
\hline REFLEXIVO-TEORICO & 40 \\
\hline REFLEXIVO-PRAGMATICO & 17 \\
\hline REFLEXIVO-TEORICO-PRAGMATICO & 5 \\
\hline ACTIVO-REFLEXIVO & 1 \\
\hline TEORICO-PRAGMATICO & 8 \\
\hline ACTIVO-TEORICO & 3 \\
\hline ACTIVO-REFLEXIVO-TEORICO-PRAGMATICO & 1 \\
\hline ACTIVO-PRAGMATICO & 6 \\
\hline ACTIVO-REFLEXIVO-PRAGMATICO & 2 \\
\hline ACTIVO-TEORICO-PRAGMATICO & 1 \\
\hline TOTAL DE ESTUDIANTES & 349 \\
\hline
\end{tabular}

Fuente: Elaboración propia. 
Gráfica 1 | Estilos de aprendizaje de los estudiantes del Colegio Unión Europea Sede A y B Grados $4^{\circ}$ a $7^{\circ}$
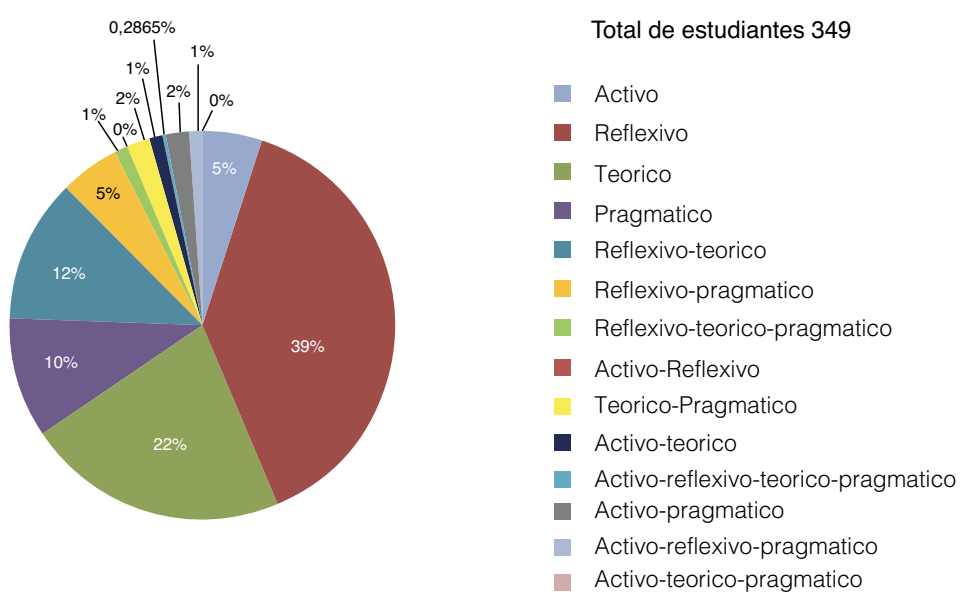

Fuente: Elaboración propia

Tabla 3 | Estilos de enseñanzas en docentes del Colegio Unión Europea Sede A y B Grados $4^{\circ}$ a $7^{\circ}$

\begin{tabular}{lc}
\hline Estilo de enseñanza & \# profesor \\
\hline ABIERTO & 2 \\
\hline FORMAL & 2 \\
\hline ESTRUCTURADO & 4 \\
\hline FUNCIONAL & 2 \\
\hline ABIERTO/ESTRUCTURADO & 1 \\
\hline ABIERTO/FORMAL/FUNCIONAL & 1 \\
\hline TOTAL DE PROFESORES & 12 \\
\hline
\end{tabular}

Fuente: Elaboración propia

En la gráfica 2 se observa que el estilo de enseñanza predominante es el estructurado con un resultado de 33\%, por el contrario, los de menor predominancia son el abiertoestructurado y abierto-formal-funcional cada uno de ellos con un resultado de $8 \%$. 
Volumen 3 • Número 1 • enero - junio 2020 • Págs. 61 - 90 •

ISSN: 2389-8798 impreso / ISSN 2539-1445 (En línea) / Tunja, Boyacá (Colombia)

Gráfica 2 | Estilos de enseñanzas en docentes del Colegio Unión Europea Sede A y B Grados $4^{\circ}$ a $7^{\circ}$

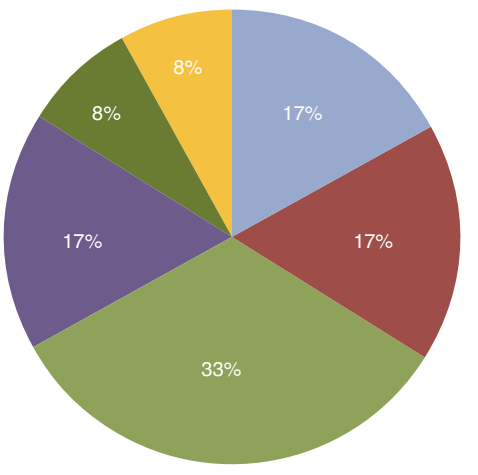

Total de profesores 12

- Abierto

Formal

Estructurado

- Funcional

- Abierto/Estructurado

- Abierto/Formal/Funcional

Fuente: Elaboración propia

En la gráfica 3 se identifica que el estilo de aprendizaje predominante es el reflexivo con un porcentaje de $58 \%$, en cambio, el de menor predominancia es el reflexivo pragmático con $3 \%$. Es importante resaltar que el estilo de enseñanza del director de grado es abierto.

Gráfica 3 | Estilos de aprendizaje grado 401

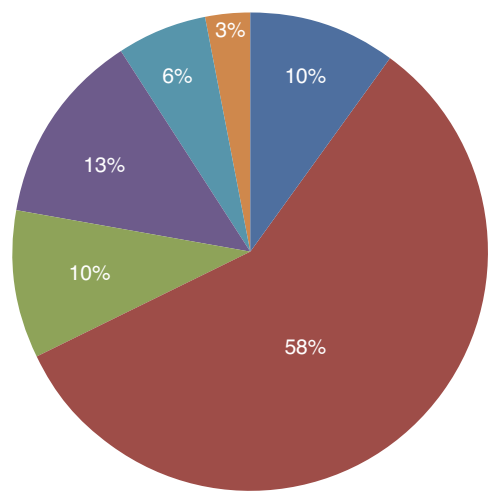

Total de estudiantes 31

- Activo

- Reflexivo

- Teorico

- Programatico

- Reflexivo/Teorico

- Reflexivo/Pragmatico

Fuente: Elaboración propia

En la gráfica 4 se refleja que el estilo de aprendizaje predominante es el reflexivo con un resultado de $40 \%$, mientras que, los de menor predominancia son pragmático, reflexivo-pragmático

巨 $\| 78$ 
y el activo, cada uno de ellos con un resultado de 3\%. Es importante resaltar que el estilo de enseñanza del director de grado es abierto, formal y funcional.

Gráfica 4 | Estilos de aprendizaje grado 402

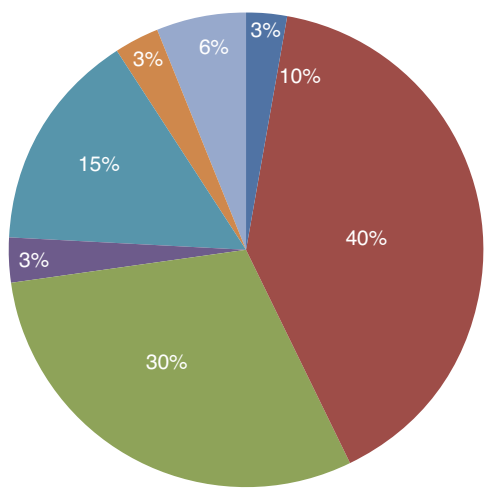

Total de estudiantes 33

Fuente: Elaboración propia

En la gráfica se evidencia que el estilo de aprendizaje predominante es el reflexivo con un resultado de $46 \%$, por el contrario, los de menor predominancia son pragmático y reflexivopragmático, cada uno de ellos con un resultado de $4 \%$. Es importante resaltar que el estilo de enseñanza del director de grado es funcional.

Gráfica 5 | Estilos de aprendizaje grado 403

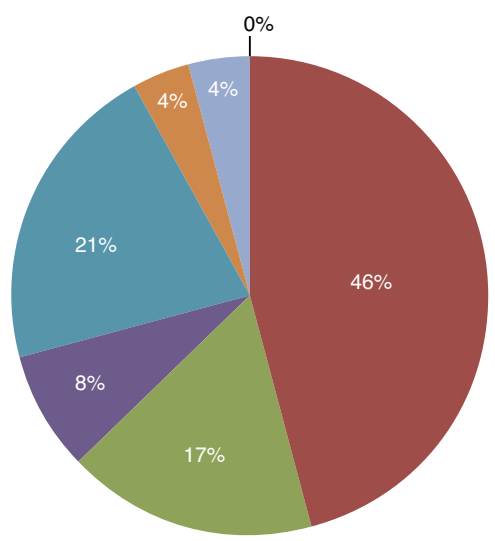

Total de estudiantes 24

- Activo

- Reflexivo

Teorico

- Programatico

- Reflexivo/Teorico

Reflexivo/Pragmatico

- Activo/Reflexivo

Fuente: Elaboración propia 
En la gráfica 6 se observa que el estilo de aprendizaje predominante es el reflexivo con un resultado de $34 \%$, en cambio, los de menor predominancia son pragmático y reflexivopragmático, cada uno de ellos con un resultado de 10\%. Es importante resaltar que el estilo de enseñanza del director de grado es el estructurado.

Gráfica 6 | Estilos de aprendizaje grado 501

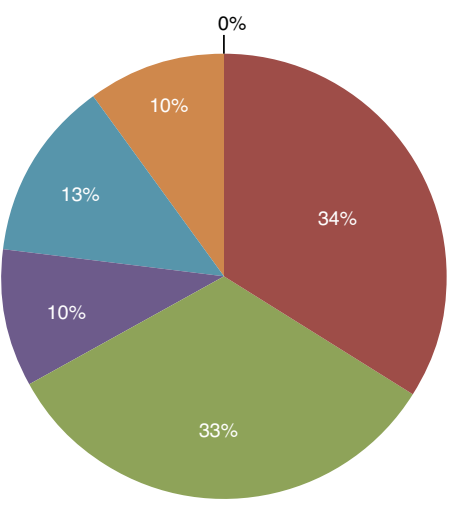

Fuente: Elaboración propia
Total de estudiantes 24

- Activo

Reflexivo

- Teorico

- Programatico

Reflexivo/Teorico

Reflexivo/Pragmatico

En la gráfica 7 se identifica que el estilo de aprendizaje predominante es el reflexivo con un resultado de $45 \%$, mientras que, los de menor predominancia son pragmático, activo y teórico-pragmático, cada uno de ellos con un resultado de $3 \%$. Es importante resaltar que el estilo de enseñanza del director de grado es funcional.

Gráfica 7 | Estilos de aprendizaje grado 502

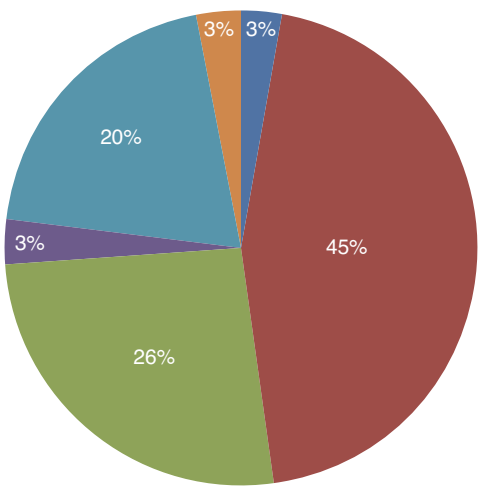

Total de estudiantes 31

$\boldsymbol{\epsilon} \mid 80$

Fuente: Elaboración propia

Activo

- Reflexivo

- Teorico

- Programatico

- Reflexivo/Teorico

Reflexivo/Pragmatico 
En la gráfica 8 se refleja que el estilo de aprendizaje predominante es el reflexivo con un resultado de $50 \%$, por el contrario, los de menor predominancia son activo y teóricopragmático, cada uno de ellos con un resultado de 3\%. Es importante resaltar que el estilo de enseñanza del director de grado es formal.

Gráfica 8| Estilos de aprendizaje grado 503

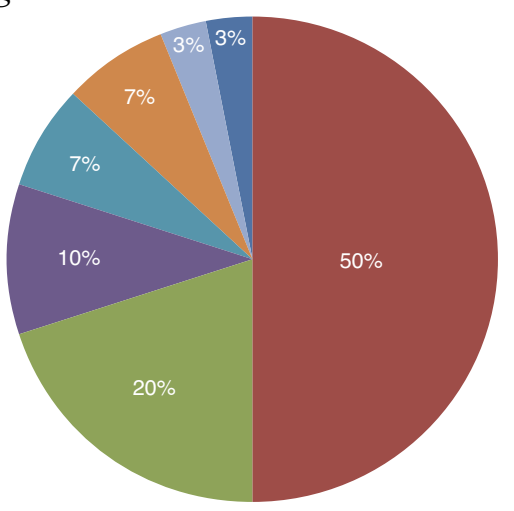

Total de estudiantes 30

- Activo

- Reflexivo

- Teorico

- Programatico

- Reflexivo/Teorico

- Reflexivo/Pragmatico

- Teorico/Pragmatico

Fuente: Elaboración propia

En la gráfica 9 se evidencia que los estilos de aprendizaje predominantes son el reflexivo y el teórico cada uno con un resultado de $31 \%$, en cambio, el de menor predominancia es el activo-teórico con un resultado de $4 \%$. Es importante resaltar que el estilo de enseñanza del director de grado es el estructurado.

Gráfica 9| Estilos de aprendizaje grado 601

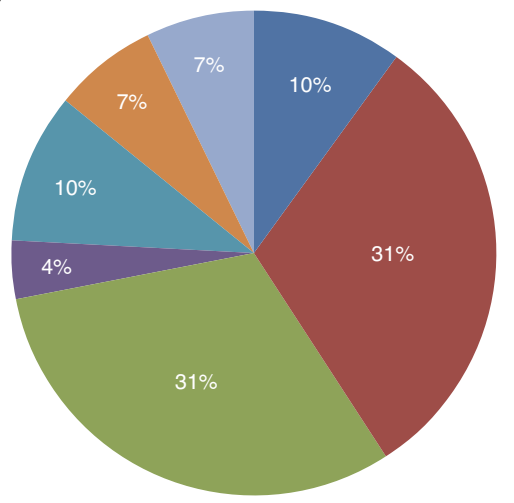

Total de estudiantes 29

Fuente: Elaboración propia

Activo

- Reflexivo

Teorico

- Activo/Teorico

- Reflexivo/Teorico

- Reflexivo/Pragmatico

Teorico/Pragmatico 
En la gráfica 10 se observa que el estilo de aprendizaje predominante es el reflexivo con un resultado de $29 \%$, mientras que, los de menor predominancia son el pragmático y el activo-reflexivo-teórico-pragmático, cada uno de ellos con un resultado de $3 \%$. Es importante resaltar que el estilo de enseñanza del director de grado es el formal.

Gráfica 10| Estilos de aprendizaje grado 602

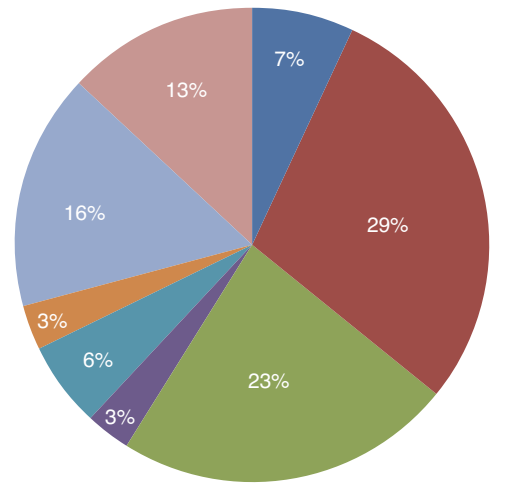

Total de estudiantes 31

- Activo

- Reflexivo

- Teorico

- Pragmatico

- Activo/Teorico

- Activo/Reflexivo/Teorico/Pragmatico

- Reflexivo/Pragmatico

- Teorico/Pragmatico

Fuente: Elaboración propia

En la gráfica 11 se identifica que el estilo de aprendizaje predominante es el reflexivo con un resultado de $35 \%$, por el contrario, los de menor predominancia son el activo-reflexivopragmático y el reflexivo-teórico-pragmático, cada uno de ellos con un resultado de $3 \%$. Es importante resaltar que el estilo de enseñanza del director de grado es el estructurado.

Gráfica 11 | Estilos de aprendizaje grado 603

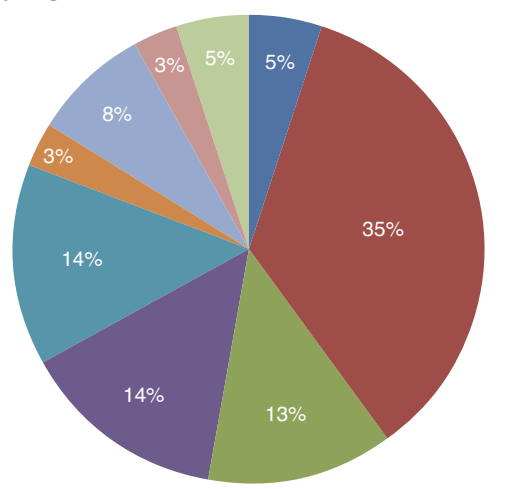

Total de estudiantes 37

$\boldsymbol{\epsilon} \mid 82$

Fuente: Elaboración propia

- Activo

- Reflexivo

- Teorico

- Pragmatico

- Reflexivo/Teorico

- Reflexivo/Teorico/Pragmatico

- Activo/Pragmatico

- Activo/Reflexivo/Pragmatico

- Reflexivo/Pragmatico 
En la gráfica 12 se refleja que el estilo de aprendizaje predominante es el reflexivo con un resultado de $42 \%$, en cambio, el de menor predominancia es el teórico con un resultado de $4 \%$. Es importante resaltar que el estilo de enseñanza del director de grado es el estructurado.

Gráfica 12 | Estilos de aprendizaje grado 701

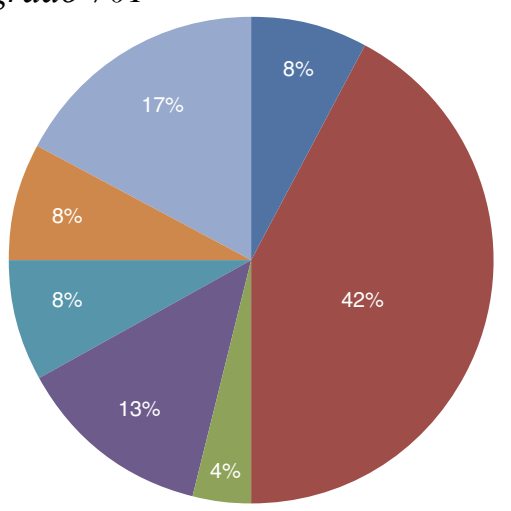

\author{
Total de estudiantes 24 \\ Activo \\ - Reflexivo \\ - Teorico \\ - Pragmatico \\ Reflexivo/Teorico \\ - Activo/Pragmatico \\ - Reflexivo/Pragmatico
}

Fuente: Elaboración propia

En la gráfica 13 se evidencia que el estilo de aprendizaje predominante es el reflexivo con un resultado de 32\%, mientras que, el de menor predominancia es el reflexivo-teóricopragmático con un resultado de $4 \%$. Es importante resaltar que el estilo de enseñanza del director de grado es abierto y estructurado.

Gráfica 13 | Estilos de aprendizaje grado 702

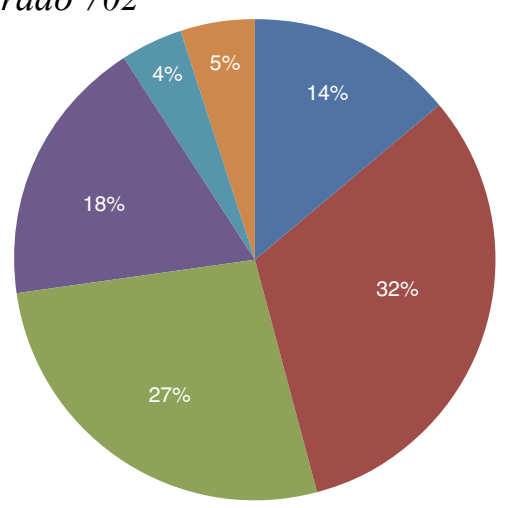

Fuente: Elaboración propia
Total de estudiantes 22

Activo

Reflexivo

- Teorico

- Pragmatico

- Reflexivo/Teorico/Pragmatico

- Reflexivo/Teorico 
En la gráfica 14 se observa que el estilo de aprendizaje predominante es el teórico con un resultado de $29 \%$, en cambio el de menor predominancia es el activo con un resultado de $3 \%$. Es importante resaltar que el estilo de enseñanza del director de grado es abierto.

Gráfica 14| Estilos de aprendizaje grado 703

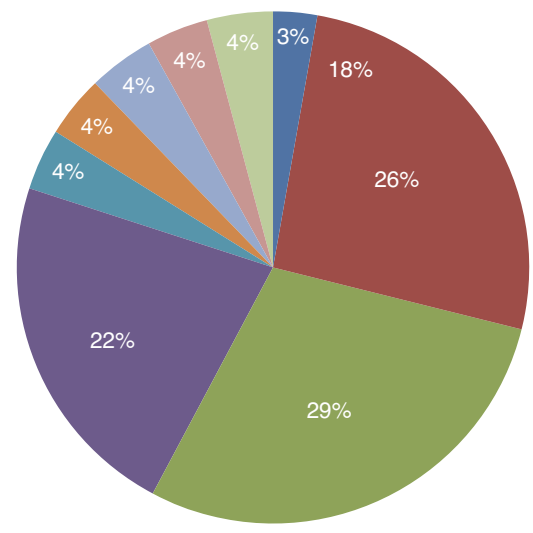

Fuente: Elaboración propia.
Total de estudiantes 27

- Activo

- Reflexivo

- Teorico

- Pragmatico

- Reflexivo/Teorico/Pragmatico

- Activo/Pragmatico

- Reflexivo/Pragmatico

- Activo/Reflexivo/Pragmatico

Activo/Teorico/Pragmatico

\section{Discusión}

Teniendo en cuenta lo planteado por Hernández \& González (2015) en relación a la importancia de conocer el entorno psicológico en el que se desarrolla el estudiante durante su proceso de aprendizaje, es necesario evaluary determinar los estilos de aprendizaje y enseñanza para favorecer las condiciones en las que se está produciendo el desarrollo y adquisición de nuevos conocimientos; y de esta manera poder prevenir problemas futuros en la educación. Dicha necesidad surge por la preocupación de incrementar la calidad educativa buscando prestar atención al mejoramiento de los procesos que se dan dentro del aula. Se resalta que esta investigación aporta elementos significativos ya que se pudo establecer la importancia de evaluar e identificar los estilos de aprendizaje de los estudiantes, lo cual permitirá concientizar sobre la importancia de la creación de ambientes de aprendizaje adecuados, que facilitarán dicho proceso y permitirá implementar nuevas estrategias metodológicas que beneficien a todos los estudiantes, teniendo en cuentas los estilos predominantes evidenciados dentro de la institución. 
Según Álvarez \& Samaniego (2015) el rendimiento académico constituye un punto de referencia que mide el grado de eficacia del proceso que se deriva de la enseñanza y el aprendizaje, logrando de esta manera definir el nivel de aprendizaje alcanzado por el alumno. Sin embargo, dicho rendimiento depende de una gran variedad de factores tanto personales, familiares y del contexto educativo en el cual se encuentra inmerso el estudiante. Por consiguiente, esta investigación reconoce la importancia de evaluar los estilos de aprendizaje y enseñanza de acuerdo al marco normativo de la educación establecida en Colombia, para de esta manera lograr identificar la influencia e incidencia de dichos estilos en el rendimiento académico de los estudiantes que favorezcan la creación de estrategias eficientes dentro del proceso educativo, que disminuyan los niveles y factores que conllevan a la deserción escolar de los alumnos.

Algunos autores como Canto, Pérez \& Plata (2016) coinciden en que los estilos de aprendizaje son aprendidos en un individuo desde etapas muy tempranas, además mencionan que existen algunas teorías que han trabajado y desarrollado el tema sobre la forma de aprender; es decir, de los estilos de aprendizaje que son aquellos rasgos cognitivos, afectivos y fisiológicos que sirven como indicadores de cómo los alumnos perciben interacciones y responden a sus ambientes de aprendizaje, relacionados con los modelos existentes sobre estilos de aprendizaje planteados en la teoría de Kolb (1984) quien menciona que este estilo se basa en los modelos de aprendizaje relacionados con la experiencia ya que es fundamental para que el individuo pueda desarrollar observaciones, análisis y reflexiones en la búsqueda de un aprendizaje significativo. Con la evaluación desarrollada se logró constatar la presencia de distintos estilos de aprendizaje presentes en las aulas de clase de la institución objeto de estudio, además cabe resaltar que existen diferencias significativas en cuanto a los estilos de enseñanza implementados por los docentes.

De acuerdo al estudio desarrollado por Bello, Almaguer \& Rodríguez (2017) donde fue aplicado el CHAEA Junior, en los resultados obtenidos se resalta el predominio que tiene el estilo de aprendizaje reflexivo en los estudiantes con un $39 \%$, y el estilo de enseñanza que predomina en los docentes es el estructurado con un $33 \%$, evidenciando que dicho estilo predominante depende de la experiencia académica del estudiante dentro de la institución. 
Por otra parte, Chiang, Larenas \& Pizarro (2016) indican que existen distintos estilos de enseñanza. El primero es el abierto que favorece a los alumnos con estilo de aprendizaje activo; el segundo es el formal que beneficia el estilo de aprendizaje reflexivo; el tercero es el estructurado que promueve el estilo teórico; y por último el estilo funcional que potencializa el aprendizaje en estudiantes con estilo pragmático. Con base a lo anterior y a los resultados obtenidos con los instrumentos de evaluación de los estilos se puede destacar que según la hipótesis planteada en esta investigación; de manera general no existe una relación significativa entre los estilos de enseñanza implementados por los docentes en el aula y los estilos propios del alumnado al momento de adquirir nuevos conocimientos que favorezcan y faciliten los procesos académicos y personales dentro de la institución. Sin embargo, en tres grados se logra evidenciar que sí existe una correlación entre el estilo de enseñanza y aprendizaje, puesto que en los grados 402, 503 y 602 el estilo de enseñanza predominante es el formal que está asociado al estilo de aprendizaje reflexivo el cual es de mayor prevalencia en la población.

\section{Conclusiones}

Con esta investigación se puede concluir que cada persona tiene características, habilidades y/o estrategias diferentes para desarrollar su proceso de aprendizaje, teniendo en cuenta que generan estilos de aprendizaje de acuerdo al estilo de enseñanza que reciben e interiorizan por medio de su historial académico y sus modelos formativos. Se logró identificar los estilos de aprendizaje y enseñanza de la población evaluada; tras la aplicación de los instrumentos CHAEA Junior y CEE; además, se logró establecer que de manera general no hay una relación significativa entre dichos estilos debido a que el estilo de aprendizaje predominante fue el reflexivo y en los docentes el estilo de enseñanza fue el estructurado lo cual se puede sustentar por medio de lo planeado por Chiang, Larenas \& Pizarro (2016).

Por otra parte, esta investigación dejó varios interrogantes en torno a los modelos tradicionales de enseñanza que se implementan en la educación colombiana debido a que es necesario que dicho modelo tenga en cuenta los diferentes estilos de aprendizaje de cada estudiante y de enseñanza de los docentes; para establecer currículos adecuados que tengan en cuenta dichas características individuales para romper el paradigma de la educación unificada y generalizada. 
Por consiguiente, es indispensable que se promuevan y realicen este tipo de estudios en colegios colombianos que favorezcan la evaluación de los estilos de aprendizaje y enseñanza antes de iniciar los procesos formativos para de esta manera identificar los diferentes estilos y así generar técnicas que permitan un entorno educativo adecuado e incluyente, permitiendo que los docentes implementen estrategias de enseñanza acordes a las características propias de los estudiantes.

Finalmente cabe resaltar que en esta investigación no se logró corroborar la hipótesis planteada inicialmente la cual consistía en una posible relación entre los estilos de enseñanza y los estilos de aprendizaje. Por tal razón se derivan otras posibles hipótesis, en primer lugar, probablemente el estilo de enseñanza no tiene un grado significativo de influencia sobre el estilo personal de aprendizaje de cada estudiante debido a que priman las experiencias y las características individuales sobre los modelos de enseñanza. En segundo lugar, la experiencia de los docentes desarrolla un modelo específico y propio que les permite planificar y ejecutar sus contenidos formativos lo cual puede impedir que ellos adopten nuevos estilos y estrategias de enseñanza. Sin embargo si se hubiera comprobado la hipótesis propuesta en esta investigación se debe tener en cuenta que conllevaría unos beneficios y/o consecuencias para los procesos educativos; dentro de los posibles beneficios estaría que los modelos de enseñanza utilizados por los docentes favorecían los procesos de aprendizaje de los estudiantes y garantizarían la adquisición de nuevos conocimientos y como posibles consecuencias se mantendría el modelo de enseñanza tradicional el cual se caracteriza por ser un modelo generalizado y en algunos casos poco incluyente.

Entre las recomendaciones finales, las instituciones deben tener en cuenta la evaluación de los estilos de aprendizaje y enseñanza como un proceso educativo que permita generar cambios significativos en los estándares de la educación tradicional para de esta manera disminuir los altos índices de bajo rendimiento académico, falta de motivación y deserción escolar.

Es importante que en las instituciones educativas se creen espacios de reflexión pedagógica que favorezcan los procesos de aprendizaje en los estudiantes y faciliten la labor del docente generando nuevas estrategias de enseñanza. 
Es necesario que los docentes reciban capacitaciones relacionadas con las didácticas inclusivas desde los estilos de aprendizaje para fortalecer los procesos formativos dentro de la institución.

Se hace indispensable que los docentes desde su labor pedagógica generen espacios de inclusión por medio de estrategias educativas que permitan favorecer el aprendizaje en el entorno escolar, generando en los estudiantes habilidades y competencias personales que potencialicen sus procesos académicos y a su vez les sirvan para enfrentar las situaciones que se presentan en su cotidianidad.

El Ministerio de Educación Nacional de Colombia debe evaluar la posibilidad de generar cambios en sus currículos académicos teniendo en cuenta que los estilos de aprendizaje y enseñanza varían según las competencias de cada estudiante y docente.

\section{Referencias}

Álvarez, M. D. V. S., \& Samaniego, M. J. G. (2015). Resultado del proceso educativo: El papel de los estilos de aprendizaje y la personalidad. Educación XX1, 18(2), 323-349.

Bello, P. H., Almaguer, R. T., \& Rodríguez, A. L. (2017). Características que presentan los estudiantes con estilos de aprendizaje diferentes en ambientes de aprendizaje colaborativo. Tendencias pedagógicas, (30), 191-206.

Bustos, G. N. (2015). Aplicaciones actuales en psicología educativa. Editorial Universitaria-Libros UDG.

Caira-Rojas, J., Urdaneta, E. M., \& Mata-Guevara, L. B. (2014). Estrategias para el aprendizaje significativo de procesos de fabricación mediante orientación constructivista. Opción, 30(75).

Chiang, M. T., Larenas, C. D., \& Pizarro, P. A. (2016). Estilos de enseñanza y aprendizaje: ¿cómo dialogan en la práctica?. Journal of Learning Styles, 9(17).

Canto, L. G. C., Pérez, E. G., \& Plata, S. J. (2016). Estilos de aprendizaje de los alumnos de la escuela superior de medicina, como estrategia de trabajo colaborativo EN EL AULA. Red Internacional de Investigadores en Competitividad, 9(1), 1656-1675.

\section{$\boldsymbol{\epsilon} \mid 88$}


Colombia. Ministerio de Educación Nacional. (2009). Ley 1290 Por la cual se reglamenta el ejercicio de la profesión de Psicología, se dicta el Código Deontológico y Bioético y otras disposiciones. Presidencia de la República de Colombia. Bogotá, Colombia, 6 de septiembre de 2006.

Delgado, J. F. S. (2014). El cuestionario Chaea-Junior o cómo diagnosticar el estilo de aprendizaje en alumnos de primaria y secundaria. Journal of Learning Styles, 7(13), 1-20.

Díaz, A.F., \& Hernández, R.G., (2015). Constructivismo y aprendizaje significativo. En: estrategias docentes para un aprendizaje significativo. México Mc Graw.

Donado, M. G. (2014). Estrategias de enseñanza en docentes y estilos de aprendizaje en estudiantes del programa de psicología de la Universidad Simón Bolívar, Barranquilla. Journal of Learning Styles, 2(3), 124-139.

Freiberg Hoffmann, A., Ledesma, R., \& Fernández Liporace, M. (2015). Estilos de aprendizaje en estudiantes universitarios ingresantes y avanzados de Buenos Aires. Liberabit, 21(1), 71-79.

García, M. A. (2016). Correlación inherente de los estilos del aprendizaje y las estrategias de enseñanza-aprendizaje. Revista Iberoamericana de Producción Académica y Gestión Educativa.1-16.

García, L. I. R., Ortiz, J. F. Z., \& Rodríguez, A. L. (2016). La relación entre los estilos de aprendizaje de los estudiantes y los estilos de enseñanza del profesor en un grupo de alumnos de primer semestre del nivel profesional. Journal of Learning Styles, 9(17), 174-205.

Greca, I., \& Moreira, M. A. (2016). Un estudio piloto sobre representaciones mentales, imágenes, proposiciones y modelos mentales respecto al concepto de campo electromagnético en alumnos de física general, estudiantes de postgrado y físicos profesionales. Investigadores en encino de ciencias, 1(1), 95-108.

Hernández Sampieri, R., Fernández Collado C., \& Baptista Lucio, M. P. (2010). Metodología de la investigación. México: Editorial Mc Graw Hill.

Hernández, M. L., \& González, M. A. (2015). La motivación en el aula: estrategia esencial para mejorar el aprendizaje en la escuela primaria. Cuadernos de Educación y Desarrollo, 55, 1-10.

Laudadío, M. J., \& Da Dalt, E. (2014). Estudio de los estilos de enseñanza y estilos de aprendizaje en la universidad. Educación y Educadores, 17(3), 483-498. 
Otero, M. R. (2016). Psicología cognitiva, representaciones mentales e investigación en enseñanza de las ciencias. Investigadores en Encino de Ciencias, 4(2), 93-119.

Peiteado, M. G. (2013). Los estilos de enseñanza y aprendizaje como soporte de la actividad docente. Journal of Learning Styles, 6(11).

Rangel, M. S. A., Ortiz, J. F. Z., \& Rodríguez, A. L. (2014). El aprendizaje de las matemáticas a través de la consideración de los estilos de aprendizaje en alumnos del nivel medio superior. Revista de Investigación Educativa de la Escuela de Graduados en Educación, 5(9), 14-20.

Renes, P., Echeverry, L. M., Chiang, M. T., \& Rangel, L. (2013). Estilos de enseñanza: un paso adelante en su conceptualización y diagnóstico. Journal of Learning Styles, 6(11), 4-18.

Rivera-Michelena, N. (2016). Una óptica constructivista en la búsqueda de soluciones pertinentes a los problemas de la enseñanza-aprendizaje. Educación Médica Superior, 30(3), 609-614.

Rodríguez-Arocho, W. (2015). Reflexividad histórica, problematización e indagación dialógica como herramientas para repensar el concepto vygotskiano de zona de desarrollo próximo. Revista Puertorriqueña de Psicología, 26(1), 10-24.

Salgado, C., Teresa, M., Díaz, L. C., \& Riva, A. A., (2013). Un cuestionario de estilos de enseñanza para el docente de Educación Superior. Revista Lasallista de Investigación, 10(2), 62-68.

Sánchez, A.L. (2016, 16 de enero) Cada año más de 300.000 niños y adolescentes abandonan el colegio. El Tiempo. Recuperado de http://www.eltiempo.com/archivo/documento/CMS-16483261, 1.

Zapata-Ros, M. (2015). Teorías y modelos sobre el aprendizaje en entornos conectados y ubicuos. Bases para un nuevo modelo teórico a partir de una visión crítica del “colectivismo". Teoría de la Educación. Educación y Cultura en la Sociedad de la Información, 16(1). 